\title{
BASIC SCIENCE ARTICLE OPEN Mesenchymal stem cells transplantation attenuates brain injury and enhances bacterial clearance in Escherichia coli meningitis in newborn rats
}

\author{
So Yoon $\mathrm{Ahn}^{1,2}$, Yun Sil Chang ${ }^{1,2,3}$, Young Eun $\mathrm{Kim}^{3}$, Se In Sung ${ }^{1}$, Dong Kyung Sung ${ }^{1,2}$ and Won Soon Park ${ }^{1,2,3}$
}

\begin{abstract}
OBJECTIVE: Neonatal meningitis caused by Escherichia coli results in significant mortality and neurological disabilities, with few effective treatments. Recently, we demonstrated that human umbilical cord blood-derived mesenchymal stem cell (hUCB-MSC) transplantation attenuated $E$. coli-induced severe pneumonia, primarily by reducing inflammation and enhancing bacterial clearance. This study aimed to determine whether intraventricular transplantation of hUCB-MSCs attenuated the brain injury in $E$. coli meningitis in newborn rats.

METHODS: Meningitis without concomitant bacteremia was induced by intraventricular injection of $5 \times 10^{2}$ colony forming units of $\mathrm{K} 1$ (-) E. coli in rats at postnatal day $(\mathrm{P}) 11$, and hUCB-MSCs $\left(1 \times 10^{5}\right)$ were transplanted intraventricularly $6 \mathrm{~h}$ after induction of meningitis. Antibiotics was started $24 \mathrm{~h}$ after modeling.

RESULT: Meningitis modeling induced robust proliferation of $E$. coli in the cerebrospinal fluid and increased mortality in rat pups, and MSC transplantation significantly reduced this bacterial growth and the mortality rate. Impaired sensorimotor function in the meningitis rats was ameliorated by MSCs injection. MSCs transplantation also attenuated meningitis caused brain injury including cerebral ventricular dilatation, brain cell death, reactive gliosis, and inflammatory response.

CONCLUSION: Intraventricular transplantation of hUCB-MSCs significantly improved survival and attenuated the brain injury via anti-inflammatory and antibacterial effects in experimental neonatal $E$. coli meningitis.
\end{abstract}

Pediatric Research (2018) 84:778-785; https://doi.org/10.1038/s41390-018-0142-5

\section{INTRODUCTION}

Bacterial meningitis is the most common and fatal infection of the central nervous system (CNS) and is a major cause of morbidity and mortality worldwide. ${ }^{1}$ Newborn infants are at higher risk because of their immature immunity and incomplete blood brain barrier (BBB). The mortality rate of neonatal bacterial meningitis varies according to the socio-economic status of the country, and ranges from 10 to $58 \%{ }^{2,3}$ Although the mortality rate has been reduced because of medical advances and the active use of antibiotic treatments, long-term neurological sequelae, which include hearing impairment, hydrocephalus, intracranial hemorrhage, mental retardation, and focal neurological deficit, have not changed and are still reported in almost $50 \%$ of survivors. ${ }^{2}$ Thus, new therapeutic methods are urgently required to improve these adverse neurological complications induced by bacterial meningitis.

Both bacterial pathogens and host inflammatory responses are known to be responsible for the pathophysiological mechanism of bacterial meningitis and the resultant brain injury and death. ${ }^{4}$ Recent preclinical and clinical studies have demonstrated that mesenchymal stem cells (MSCs) transplantation protected against neonatal intractable disorders including bronchopulmonary dysplasia, ${ }^{5-7}$ hypoxic ischemic encephalopathy, ${ }^{8,9}$ neonatal stroke, $^{10,11}$ and severe intraventricular hemorrhage, ${ }^{12}$ via their paracrine anti-inflammatory, antiapoptotic, and antioxidative effects, as well as enhancing the regeneration of the injured host tissue by secreting various growth factors.

Furthermore, recent studies have demonstrated that MSCs not only attenuated inflammatory responses, but also enhanced bacterial clearance in bacterial pneumonia and/or sepsis. ${ }^{13,14}$ These findings suggested that MSCs could be a promising novel adjuvant therapeutic modality in addition to antibiotics for various infectious disorders, including bacterial pneumonia, sepsis, and meningitis. However, the therapeutic efficacy of MSC transplantation to attenuate brain jury in neonatal bacterial meningitis has not been tested. Therefore, favorable experimental results showing that transplantation of MSCs could protect against bacterial meningitis in a newborn animal model would be necessary for future successful clinical translation in the treatment of neonatal bacterial meningitis patients.

Thus, in the present study, we aimed to determine the therapeutic efficacy of the transplantation of MSCs to improve survival and attenuate brain injuries using our recently developed newborn rat model of Escherichia coli meningitis without concomitant bacteremia, and to assess whether the protective mechanism is related to the anti-inflammatory and antibacterial effects of the MSCs. As simultaneous systemic bacteremia aggravates and confounds the mortality and brain injury induced

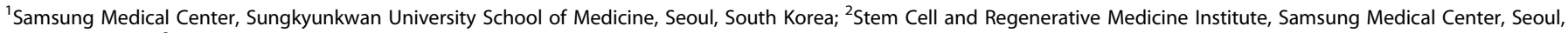
South Korea and ${ }^{3}$ Department of Health Sciences and Technology, SAIHST, Sungkyunkwan University, Seoul, South Korea

Correspondence: Won Soon. Park (wonspark@skku.edu, ws123.park@samsung.com)

These authors equally contributed this work: So Yoon Ahn, Yun Sil Chang
}

Received: 30 January 2018 Revised: 26 July 2018 Accepted: 27 July 2018

Published online: 14 August 2018 
by meningitis, we used $\mathrm{K} 1$ capsule negative $E$. coli to confine the infection to the CNS without concomitant systemic bacteremia, ${ }^{4,15}$ and inoculated the bacteria intraventricularly to simulate the neuropathological progression of clinical neonatal bacterial meningitis, beginning with ventriculitis. ${ }^{16,17}$ We transplanted human umbilical cord blood (UCB)-derived MSCs intraventricularly because of their ease of isolation, lower immunogenicity, higher proliferation capacity, and better paracrine potency and therapeutic efficacy compared with MSCs derived from adult tissues, such as adipose tissue- or bone marrow-derived MSCs. ${ }^{18}$ The therapeutic efficacy of MSC transplantation was evaluated using brain magnetic resonance imaging (MRI) in vivo; negative geotaxis tests; assessment of inflammatory cytokines, such as interleukin (IL)-1 a, IL-1ß, IL-6, and tumor necrosis factor (TNF)-a; and histology in the periventricular area and hippocampus by glial fibrillary acidic protein (GFAP), reactive microglial marker (ED-1), and terminal deoxynucleotidyl transferase nick end labeling (TUNEL) staining.

\section{METHODS}

Cell preparation

UCB-derived MSCs were obtained from Medipost Co., Ltd. (Seoul, Korea). ${ }^{18,19}$ Characterization of MSCs were previously described. ${ }^{19}$ MSCs from a single donor at passage six were used in this study.

\section{Bacterial preparation}

We used $E$. coli as the source of infection because it is a leading cause of bacterial meningitis in newborn infants. ${ }^{20}$ EC5ME, an un-encapsulated mutant strain of $E$. coli possessing the $\mathrm{K} 1$ capsular polysaccharide C5 (serotype 018:K1:H7) (a kind gift of Professor Kwang Sik Kim, Johns Hopkins University, MD) ${ }^{4,15}$ was used to induce only bacterial meningitis, but not secondary bacteremia, in this study. Bacteria were cultured overnight in 10 $\mathrm{mL}$ of brain heart infusion broth (BHI, BD Difco, Sparks, MD) at $37^{\circ} \mathrm{C}$. The bacteria were then diluted in $\mathrm{BHI}$ medium and grown for $1 \mathrm{~h}$ to the mid-logarithmic phase. The suspension was centrifuged for $10 \mathrm{~min}$ at $5000 \times \mathrm{g}$ and washed with normal saline. Using a spectrophotometer, the optical density of bacteria samples was measured and adjusted to the desired concentration. The final E. coli preparation contained $5 \times 10^{2}$ colony forming units (CFU) in $10 \mu \mathrm{l}$ of normal saline. The accuracy of the inoculum size was confirmed by serial dilution, overnight culture on blood agar plates, followed by colony counting to determine the CFU.

\section{Animal model}

The experimental protocols described herein were reviewed and approved by the Institutional Animal Care and Use Committee (IACUC) of Sungkyunkwan University School of Medicine (SUSM). SUSM is an Association for Assessment and Accreditation of Laboratory Animal Care International (AAALAC International) accredited facility and abides by the Institute of Laboratory Animal Resources (ILAR) guidelines.

Supplemental Figure S1 shows details of the experimental schedule. The experiment began at postnatal day (P) 11 and continued through P17. To induce meningitis, newborn SpragueDawley rats (Orient Co, Seoul, Korea) were anesthetized using 2\% isoflurane in oxygen enriched air, and $5 \times 10^{2}$ CFU of EC5ME inoculum in $10 \mu \mathrm{l}$ of normal saline were slowly infused into the left ventricle under stereotactic guidance (Digital Stereotaxic Instrument with Fine Drive, MyNeurolab, St. Louis, MO; coordinates: $x=-0.5 \mathrm{~mm}, y=+1.3 \mathrm{~mm}, z=+3.0 \mathrm{~mm}$ relative to the bregma) at P11. For the normal control group (NC), an equal volume of normal saline was given intraventricularly.

For cell transplantation, $1 \times 10^{5}$ human UCB-derived MSCs in 10 $\mu \mathrm{l}$ of normal saline for meningitis with MSC transplantation group (MT) or identical volume of normal saline alone for the meningitis control group (MC) or NC into the right ventricle under stereotactic guidance $(x=+0.5 \mathrm{~mm}, y=+1.3 \mathrm{~mm}, z=+3.0$ $\mathrm{mm}$ relative to the bregma) at $6 \mathrm{~h}$ after induction of meningitis. After the procedure, rat pups were allowed to recover and then returned to their dams; there was no mortality associated with the procedures.

Antibiotics (ampicillin, delivered intraperitoneally, $200 \mathrm{mg} / \mathrm{kg} /$ day), started $24 \mathrm{~h}$ after the induction of meningitis, were administered for three consecutive days to all rats with induced meningitis (MC and MT). First, 10 rat pups in each group were allocated to assess the acute pathophysiological changes, and the survivors were sacrificed at $24 \mathrm{~h}$ (P12) after bacterial inoculation for histopathological assessment (all $n=5$ for NC, MC, and MT) and biochemical analyses (all $n=5$ for NC, MC, and MT) at P12. We also conducted a time course experiment in animals for each group ( $n=20,20$, and 18 for NC, MC, and MT, respectively) to determine the survival rate until sacrifice of the survivors at P17 for histopathological assessment $(n=10,7$, and 8 for NC, MC, and MT, respectively) and biochemical analyses $(n=10,6$, and 9 for NC, MC, and MT, respectively).

Cerebrospinal fluid (CSF) and blood were obtained to determine the bacterial titer at $6 \mathrm{~h}, 24 \mathrm{~h}$, and 6 days (P17) after bacterial inoculation. Brain MRI was performed at P17. All animals were weighed daily and were euthanized at P12 and P17 under deep pentobarbital anesthesia ( $60 \mathrm{mg} / \mathrm{kg}$, intraperitoneal). Whole brain tissue and CSF samples were prepared as described in the Supplementary Material (online).

\section{Bacterial quantification}

Bacterial concentrations from each study group were measured in the CSF and blood at $6 \mathrm{~h}, 24 \mathrm{~h}$, and 5 days after bacterial inoculation for the induction of meningitis. Bacteria CFU in the CSF and blood were measured at dilutions of $10^{-4}-10^{-8}$ plated on $\mathrm{BHI}$ agar after overnight incubation at $37^{\circ} \mathrm{C}$, followed by colony counting.

In vivo MRI and assessment of the volume ratio of brain Injury Brain MRI was performed on neonatal rats at P17. MRI was performed using a 7.0-Tesla MRI system (Bruker-Biospin, Fällanden, Switzerland) as described previously ${ }^{10,12}$ and in the Supplementary Material (online). To determine the extent of meningitis-induced brain injury, the ratio of the lateral ventricles or parenchymal infarction area to the whole brain volume was calculated for each pup. Independent examiners, who were blinded to the experimental groups, calculated the volume ratio by manually outlining the ventricles, parenchymal infarction area, and the whole brain in $12 \mathrm{MRI}$ slices, using ParaVision software (version 2.0.2, Bruker, BioSpin, Karlsruhe, Germany), as previously described. ${ }^{10}$ Volume estimates were then made according to Cavalieri's principal.

\section{Functional behavioral tests}

To evaluate the motor coordination of newborn rat pups, negative geotaxis test which assess innate postural response appears as early as the second week of life in the normal pups ${ }^{21}$ were performed at P12, P14, and P17, as described in the Supplementary Material (online). The time required for pups to rotate $180^{\circ}$ to face uphill after release on a slanted slope was recorded. The values were analyzed by date, and the average time from three trials was used as the final result. Behavioral tests were conducted by two independent evaluators who were blinded to the treatments of the groups.

TUNEL assay

Cell death in the periventricular white matter and dentate gyrus was assessed using the immunofluorescent TUNEL technique (kit S7110 ApopTag, Chemicon, Temecula, CA). A blinded evaluator counted the TUNEL-positive nuclei in the periventricular area, 


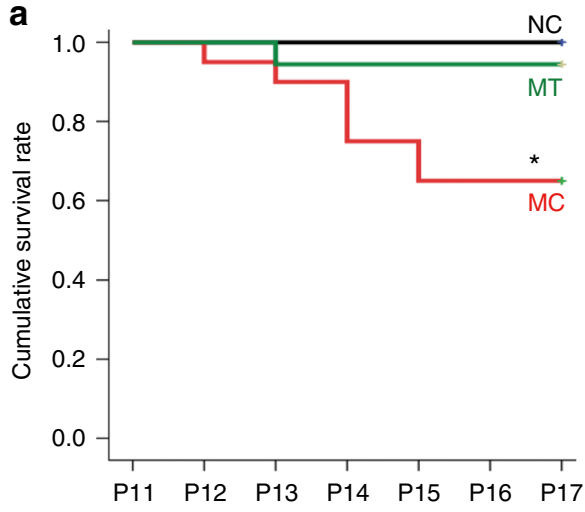

b

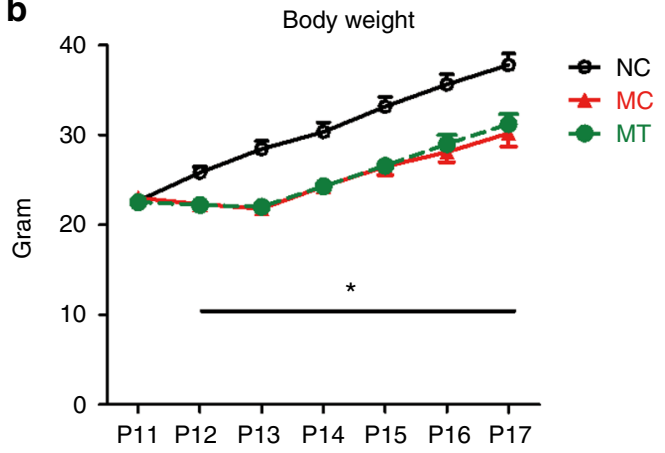

Fig. 1 Survival rate and body weight growth of the experimental animals, $\mathrm{P}$ postnatal day, NC normal control rats, $\mathrm{MC}$ meningitis control rats, MT meningitis with human umbilical cord blood (UCB)derived mesenchymal stem cells (MSC) transplantation

including the corpus callosum and caudate nucleus; and the hippocampal area, including the dentate gyrus, in the coronal brain sections. Three coronal sections $(+0.95 \mathrm{~mm}$ to $-0.11 \mathrm{~mm} /$ Bregma) from each brain and three random non-overlapping fields from each section were selected and evaluated.

Immunohistochemistry

Immunohistochemical analysis of gliosis (neuronal specific GFAP and reactive microglia (ED-1)) was performed on deparaffinized 4$\mu \mathrm{m}$ thick brain sections, as previously reported. ${ }^{12}$ The immunofluorescent GFAP or ED-1 staining intensity was evaluated in in the periventricular area, including the corpus callosum and caudate nucleus; and the hippocampal area, including the dentate gyrus, in the brain coronal sections by a blinded observer using Image $J$ software (National Institutes of Health). Three coronal sections $(+0.95 \mathrm{~mm}$ to $-0.11 \mathrm{~mm} /$ Bregma) were stained from each brain and three random non-overlapping fields were assessed from each section.

Enzyme-linked immunosorbent assay

IL-1a, IL-1 $\beta$, IL-6, and TNF-a concentrations in brain tissue homogenates at P12 and P17 were measured using a Milliplex MAP ELISA Kit according to the manufacturer's protocol (Millipore, Billerica, MA).

\section{Statistical analyses}

Data are expressed as the mean \pm the standard error of the mean. For continuous variables with a normal distribution, statistical comparison between groups MC and MT was performed using Student's $t$ test or the Mann-Whitney test. All data were analyzed using SPSS version 18.0 (IBM, Chicago, IL). $P<0.05$ was considered statistically significant.

\section{RESULTS}

Survival and body weight

To determine the beneficial effects of MSCs against meningitis, the survival rate and body weight gain in each group were monitored throughout the experiment. While the reduced survival rate in the MC group (65\%) compared with that in the NC group (100\%) was significantly improved in the MT group (94\%), the reduced weight gain observed in the $M C$ group compared with that in the NC group was not significantly improved in the MT group (Fig. 1a, b).

\section{Bacterial counts}

To evaluate whether MSCs reduced the meningitis-induced bacterial burdens, CFU were counted in the CSF and blood from each study group at $6 \mathrm{~h}$ (P11), $24 \mathrm{~h}$ (P12), and 6 days (P17) after the induction of meningitis. No bacterial growth in the blood was detected in all study groups throughout the experiment. While the bacterial counts in the CSF at $6 \mathrm{~h}$ after the induction of meningitis were not significantly different between the MC and MT groups, CSF bacterial counts in the MT group were significantly lower compared with those in the MC group at $24 \mathrm{~h}$ after the induction of meningitis (Fig. 2a). No bacterial growth in the CSF was detected in all study groups at 6 days after the induction of meningitis.

\section{Brain MRI}

To assess whether MSCs attenuated the extent of meningitisinduced brain infarction and hydrocephalus, in vivo brain MRI was performed, and the degree of brain infarct in the cortex and the dilatation of ventricles in the whole brain, as evidenced by the hyperintense areas in T2-weighted MRI performed at P17, were measured. The increased ventriculomegaly volume ratio observed in the MC group was significantly improved in the MT group at P17 (Fig. 2b, c). However, the increased brain infarct volume ratio observed in the MC group was not significantly attenuated in the MT group (Fig. 2b, c). The gross brain injury detected in brain MRI was also observed in gross brain histologic section (Supplemental figure S2).

\section{Functional behavior test}

To assess whether MSCs improved the meningitis-induced impaired sensorimotor function, negative geotaxis tests were performed serially at $\mathrm{P} 12, \mathrm{P} 14$, and $\mathrm{P} 17$. In the negative geotaxis test, the significant functional impairment observed in the $M C$ group compared with that in the NC group was significantly improved in the MT group at P12 and P14 (Fig. 3). At P17, no significant difference in negative geotaxis performance was observed between the study groups.

Cell death and reactive gliosis

To determine whether MSCs attenuated meningitis-induced brain cell death and reactive gliosis, the number of TUNEL-positive cells and the density of GFAP-positive cells in the periventricular area and hippocampal area were assessed at P17 using immunohistochemistry. The increased number of TUNEL-positive cells and density of GFAP-positive cells in both the periventricular area and hippocampus observed in the MC group compared with those in the NC group were significantly improved in the MT group (Figs. 4, 5 and Supplemental figure S3).

Brain inflammation

To determine whether MSCs attenuated the brain inflammation induced by meningitis, we analyzed inflammatory cytokine concentrations, including IL-1a, IL-1 $\beta, \mathrm{IL}-6$, and TNF- $\alpha$ in the whole brain tissue homogenates at P12 and P17. We also assessed the active brain macrophages, as evidenced by ED-1 positive cells in the periventricular area and hippocampal area, by immunohistochemistry at P17. Increased levels of inflammatory cytokines and 
a

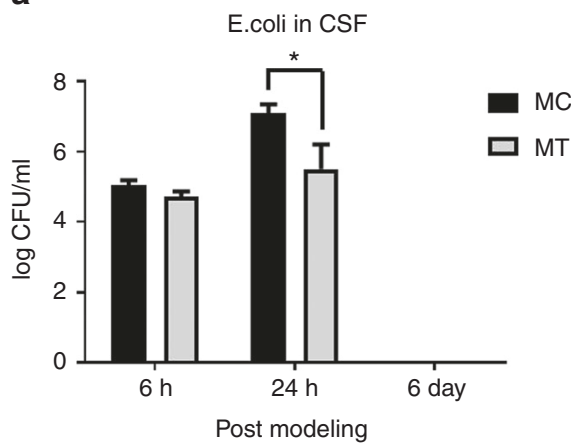

C Volume ratio of injury area to whole brain

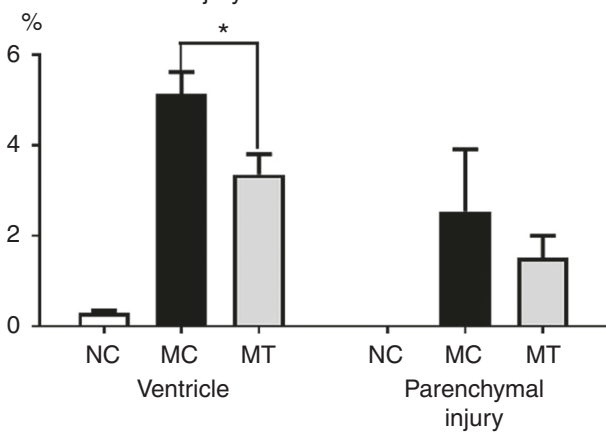

b

NS
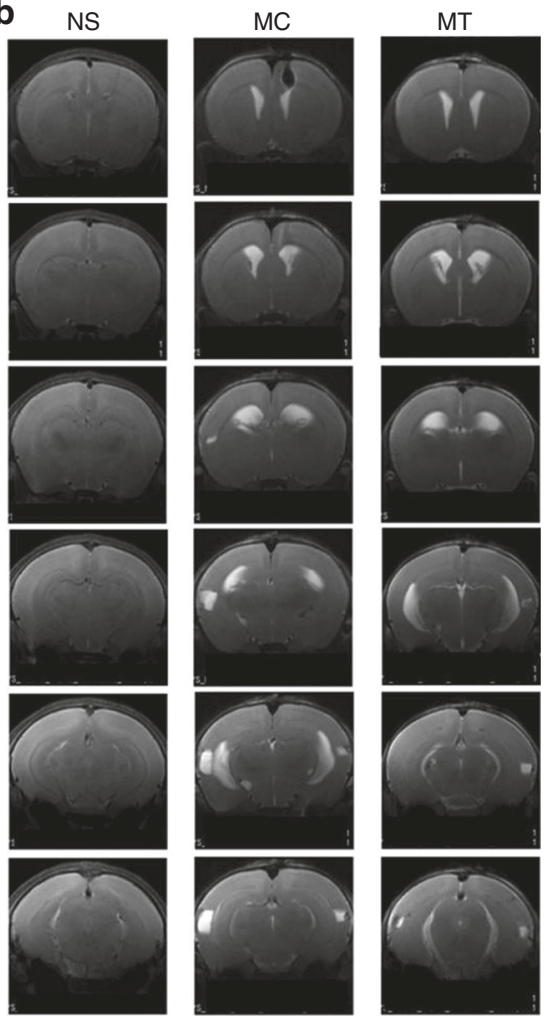

Fig. 2 Stem cell transplantation attenuated Escherichia coli proliferation in the cerebrospinal fluid (CSF) and reduced ventricular dilatation and after E. coli-induced meningitis. a CFU of cultured E. coli in the CSF at $6 \mathrm{~h}$ and $24 \mathrm{~h}$ after modeling. b Representative serial brain magnetic resonance imaging (MRI) results from each group at 6 days after inducing meningitis (P17). c The ventricle or parenchymal infarct area to whole brain volume ratio, as measured by MRI. Data are expressed as mean \pm SEM. P postnatal day, NC normal control rats, MC meningitis control rats, MT meningitis with human umbilical cord blood (UCB)-derived mesenchymal stem cell (MSC) transplantation.* $P<0.05$ vs. MC

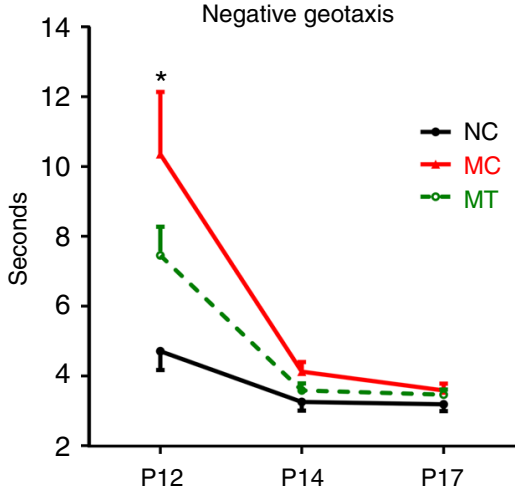

Fig. 3 Sensorimotor functional outcomes on the negative geotaxis test. Data are expressed as mean \pm SEM. P postnatal day, NC normal control rats, MC meningitis control rats, MT meningitis with human umbilical cord blood (UCB)-derived mesenchymal stem cell (MSC) transplantation. ${ }^{*} P<0.05$ vs. MC

numbers of ED-1-positive cells were observed in MC group compared with those in the NC group were significantly attenuated in the MT group (Figs. 4-6).

\section{DISCUSSION}

Developing an appropriate animal model that simulates clinical neonatal bacterial meningitis is an essential first step to develop new therapeutic modalities for this intractable and devastating disorder. Bacteremia meningitis newborn rat model is associated with high mortality and difficult to induce significant neuropathological abnormalities. Furthermore, although brain injury primarily results from local meningeal infection, concomitant systemic bacteremia might aggravate meningitis-induced disease severity and mortality. Due to this discordance between disease severity and brain injury, in this study, we tried to develop animal modal of neonatal meningitis that could dissect the role of local meningeal infection and systemic bacteremia. This might be essential for evaluating the pathophysiologic mechanism of brain injury induced solely by meningitis, and for testing the therapeutic efficacy of many new treatment approaches to reduce the meningitis-induced brain injuries. In this study, we used our recently developed newborn rat model of neonatal meningitis at $\mathrm{P} 11$, which is comparable to the human brain at birth. ${ }^{22}$ To induce meningitis, $\mathrm{K} 1$ capsule-negative $E$. coli, the most common gramnegative pathogen, was used to prevent the confounding aggravation of mortality and brain injury by concomitant bacteremia. An EC5ME inoculum dose of $5 \times 10^{2}$ CFU was injected intraventricularly to simulate clinical neonatal meningitis. After the induction of meningitis, our data showed clinically relevant mortality of $35 \%$, ${ }^{23}$ cerebral infarct and hydrocephalus by brain MRI, impaired behavioral tests and inflammatory responses, and ensuing histological abnormalities, which indicated that our newborn rat pup model is suitable and appropriate to study neonatal bacterial meningitis and to test new treatments.

Neonatal bacterial meningitis is still associated with alarmingly high rates of mortality and morbidity, despite appropriate antimicrobial therapy. ${ }^{23}$ In addition, the evolution of antibiotic resistance further complicates treatment options. To overcome this problem, new therapeutic strategies with pleotropic protective effects, including reduction of the bacterial load and attenuation of inflammatory responses, would be necessary. Recent studies demonstrated that MSC transplantation 


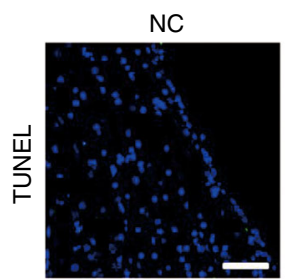

Periventriclular area
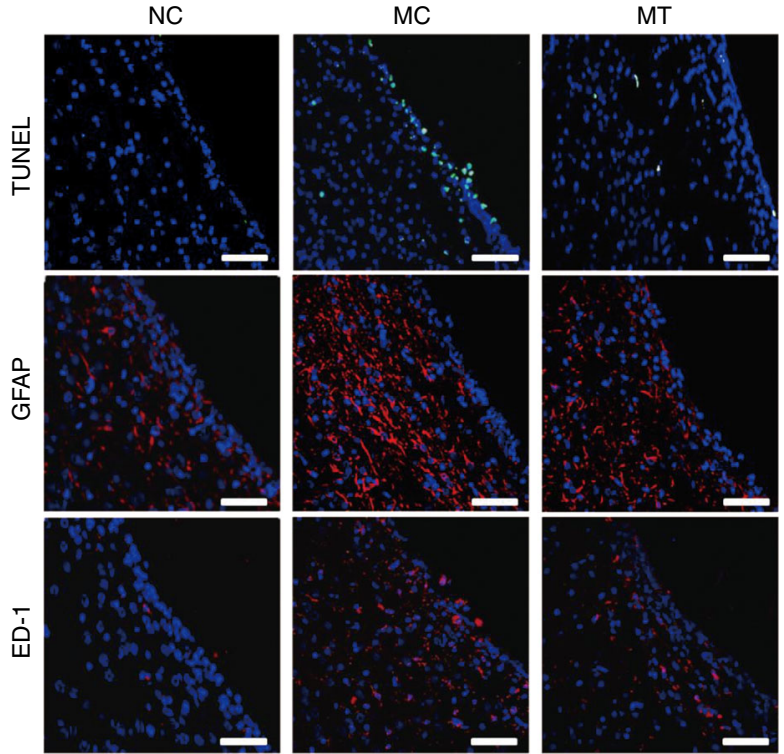

b
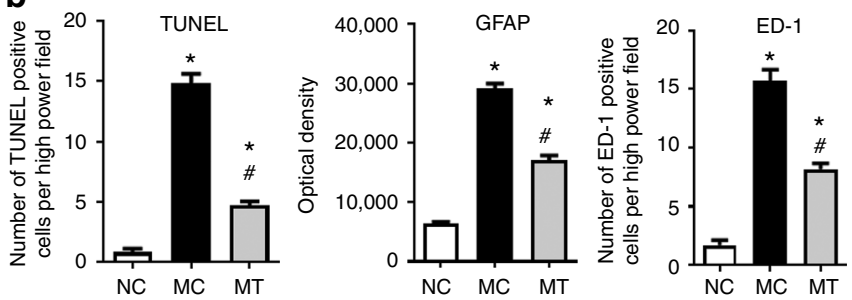

Fig. 4 In the periventricular area, stem cell transplantation ameliorated the increased cell death, reactive gliosis, and activated macrophages induced by severe meningitis. a Representative immunofluorescence photomicrographs of the periventricular area and hippocampal area with staining for terminal deoxynucleotidyl transferase nick end labeling (TUNEL) (green), glial fibrillary acidic protein (GFAP) (red), ED-1 (ectodysplasin A, red), and 2-(4amidinophenyl)-1H-indole-6-carboxamidine (DAPI; (blue) (original magnification; $\times 200$, scale bars; $25 \mu \mathrm{m}$ ). b Average number of TUNEL-positive cells and ED-1 positive cells, and average light intensity of GFAP staining in the periventricular area. Data are expressed as mean \pm SEM. P postnatal day, NC normal control rats, MC meningitis control rats, MT meningitis with human umbilical cord blood (UCB)-derived mesenchymal stem cell (MSC) transplantation.* $P<0.05$ vs. NC, \#P<0.05 vs. MC

significantly attenuated severe pneumonia and/or sepsis via their anti-inflammatory and antibacterial activities. ${ }^{14,24}$ In the present study, intraventricular transplantation of human UCB-derived MSCs coupled with antibiotic treatment significantly reduced CSF bacterial titers, improved meningitis-induced mortality, impaired behavioral tests, inflammatory responses, and the resultant brain injuries compared with antibiotic treatment alone. Overall, these findings strongly support the potential use of human UCB-derived MSCs as a new and effective adjuvant treatment in addition to antibiotics for neonatal bacterial meningitis.

Both the degree of bacterial infection and inflammation are strongly associated with adverse outcomes, such as mortality and neurological morbidities, of neonatal bacterial meningitis. Our data showing reduced CSF bacterial concentration, reduced proinflammatory cytokines (such as IL-1a, IL-1 $\beta$, IL- 6 , and TNF- $a$ ), and ED-1 positive cells after human UCB-derived MSC transplantation indicated that their protective effects against neonatal bacterial meningitis are mediated through their antibacterial and anti- a
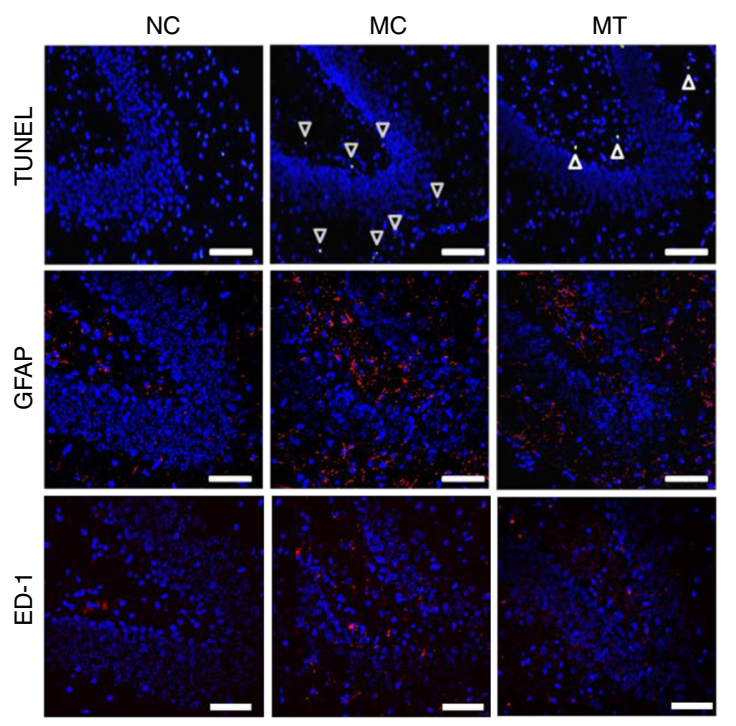

b
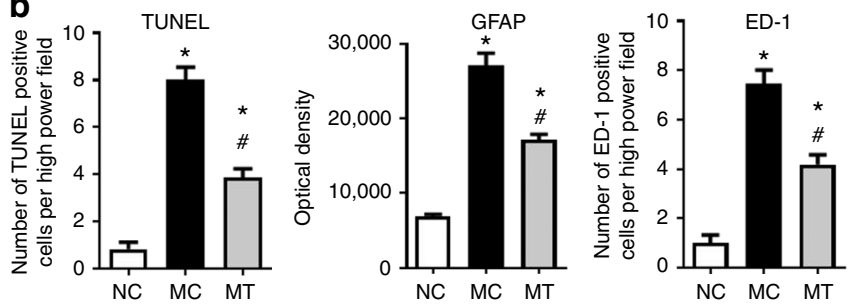

Fig. 5 In the hippocampal area, stem cell transplantation ameliorated the increased cell death, reactive gliosis, and activated macrophages induced by severe meningitis. a Representative immunofluorescence photomicrographs of the periventricular area and hippocampal area with staining for terminal deoxynucleotidyl transferase nick end labeling (TUNEL) (green), glial fibrillary acidic protein (GFAP) (red), ED-1 (ectodysplasin A, red), and 2-(4amidinophenyl)-1H-indole-6-carboxamidine (DAPI; (blue) (original magnification; $\times 200$, scale bars; $25 \mu \mathrm{m}$ ). b Average number of TUNEL-positive cells and ED-1 positive, cells and the average light intensity of GFAP staining in the periventricular area. Data are expressed as mean \pm SEM. P postnatal day, NC normal control rats, MC meningitis control rats, MT meningitis with human umbilical cord blood (UCB)-derived mesenchymal stem cell (MSC) transplantation.* $P<0.05$ vs. NC, \# $P<0.05$ vs. MC

inflammatory effects. In our previous study, we confirmed the antibacterial effects of MSCs against E.coli, and microarray analysis revealed a significant up-regulation of toll-like receptor (TLR)-2, TLR-4, and $\beta$-defensin 2 in MSCs when compared to fibroblasts after $E$. coli exposure. Moreover, this study showed that $\beta$-defensin 2 was involved in the antibacterial effects of MSCs, which were mediated by TLR-4. Thus, as a next step, the $\beta$-defensin- and TLR4-mediated antibacterial effects of MSCs should be evaluated in the E.coli-induced neonatal meningitis model in a future study. In addition, inhibiting anti-inflammatory pathway using siRNA might be helpful to determine the protective mechanism. There is no doubt that meticulously designed study should be required to prove detailed mechanism of MSCs in this neonatal bacterial meningitis model.

Recently, we demonstrated that TLR-4 in the transplanted MSCs played a seminal role in attenuating $E$. coli-induced severe pneumonia, by both the microbicidal effects and antiinflammatory effects of the MSCs. ${ }^{14}$ However, considering the pro-inflammatory effects of TLR-4-primed $\mathrm{MSCs}^{25}$ the contradictory results showing that TLR-4 from MSCs exerts antiinflammatory effects in $E$. coli-induced pneumonia are difficult to 

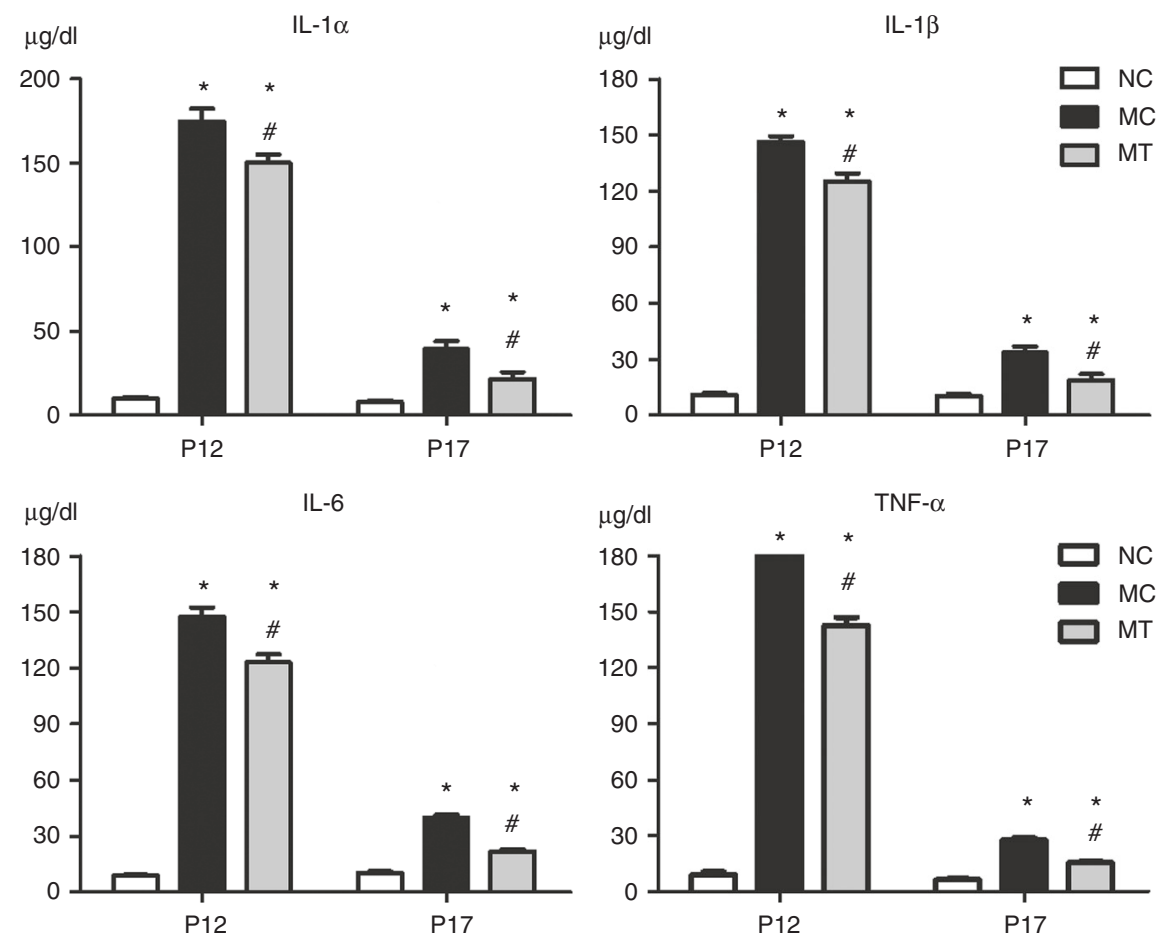

Fig. 6 Inflammatory cytokine (interleukin (IL)- $1 \alpha$, IL- $\beta$, IL-6, and tumor necrosis factor (TNF)- $\alpha$ ) concentrations in brain homogenates at P12 and P17. Data are expressed as mean \pm SEM. P postnatal day, NC normal control rats, MC meningitis control rats, MT meningitis with human umbilical cord blood (UCB)-derived mesenchymal stem cell (MSC) transplantation. ${ }^{*} P<0.05$ vs. NC, \# $P<0.05$ vs. MC

explain. Overall, these findings suggested that transplanted MSCs sense and control host inflammation by switching between their dual roles as pro-inflammatory mediators to eliminate pathogens by augmenting antimicrobial immunity, and as anti-inflammatory mediators to attenuate infection-induced host tissue injuries. Further studies are necessary to clarify this.

The protective effects are primarily mediated in a paracrine manner rather than by direct regeneration of transplanted MSCs. ${ }^{26}$ Various paracrine factors secreted by transplanted MSCs, including vascular growth factor and hepatocyte growth factor for bronchopulmonary dysplasia; ${ }^{27}$ brain-derived neurotrophic factor for intraventricular hemorrhage; ${ }^{26}$ TLR-4 signaling for $E$. coli pneumonia; ${ }^{14}$ and transforming growth factor- $\beta, \mathrm{IL}-10, \mathrm{IL}-13$, and TNF-a stimulated gene/protein $6^{28}$ for sepsis; have been demonstrated to mediate their anti-inflammatory effects. In addition, keratinocyte growth factor, ${ }^{29} \mathrm{LL}-37,{ }^{24}$ lipocalin $2{ }^{30}$ and beta defensin $2^{14}$ for pneumonia and/or sepsis have been demonstrated to mediate their antibacterial effects. Further studies are necessary to identify the paracrine factors secreted from the transplanted MSCs that mediate their protective anti-inflammatory and antibacterial effects in neonatal bacterial meningitis.

Besides of bacteria, bacterial toxins are also believed to play an important role in brain injury after bacterial meningitis. Recent study presented that exposure of MSCs to LPS (lipopolysaccharides) augmented their trophic effects and functional properties to defend against the harsh inflammatory environment. ${ }^{31}$ Given this finding, in the E.coli meningitis environment, the transplanted donor MSCs might be stimulated by LPS and further studies would be required to prove this.

Brain imaging in adults during the acute phase of bacterial meningitis demonstrated increased ventriculomegaly associated with increased mortality. ${ }^{32}$ In agreement with the clinical findings, ${ }^{17,32}$ the extent of ventriculomegaly and cerebral infarct was bacterial inoculum dose-dependent in our previously developed newborn rat model of neonatal meningitis (data not shown). In the present study, although cerebral infarct after induction of experimental meningitis was not significantly improved, the extent of ventriculomegaly was significantly improved after human UCB-derived MSC transplantation. Taken together, these findings suggested that in addition to being an early prognostic indicator, brain MRI could also be useful to assess the therapeutic efficacy of any new adjuvant treatments, including stem cell transplantation, in both clinical and experimental settings of meningitis. ${ }^{17,32}$

Brain injuries observed in experimental models of neonatal meningitis are unique in consistently reproducing both hippocampal damage and cortical necrosis. ${ }^{33,34}$ In the present study, the extent of inflammatory responses, and the increased number of TUNEL, GFAP, and ED-1 positive cells in the hippocampus and periventricular area, were improved significantly using human UCB-derived MSC transplantation. Inflammatory responses are primarily responsible for the ensuing brain injury in bacterial meningitis; ${ }^{15,33,34}$ therefore, our data showing improved meningitis-induced brain injury using MSC transplantation might be primarily mediated by their anti-inflammatory effects, in addition to their antiapoptotic effects.

Besides attenuating meningitis-induced inflammatory responses and the ensuing brain injuries, improving the functional outcome is important for the clinical application of MSC transplantation for neonatal bacterial meningitis. In rats, the negative geotaxis test, which is the postural reflex to face uphill on a slanted slope, has been known to develop in the second week of life. ${ }^{35}$ In the present study, meningitis-induced impaired performance in the negative geotaxis test was improved significantly by MSC transplantation, even at P12 and P14. No significant difference between the meningitis control and MSC transplantation groups was observed at P17, which might be attributable to the high mortality of the most injured rats in the meningitis control group.

Determining the optimal route of MSC transplantation is essential to translate these beneficial experimental results into clinical trials. In this study, human UCB-derived MSCs were 
transplanted intraventricularly, because the neuropathology of neonatal bacterial meningitis begins with choroid plexitis and ventriculitis $s^{36,37}$ and progresses to arachnoditis and vasculitis, leading to brain edema, hydrocephalus, infarction, and periventricular leukomalacia. ${ }^{38}$ Local intraventricular administration of MSCs is therapeutically more effective than their systemic intravenous delivery. ${ }^{39}$ Furthermore, no additional surgical procedures are necessary, unlike in adults, because the anterior fontanel is open in newborn infants. As CSF circulates, more invasive intraventricular transplantation might be replaced with less invasive intraspinal transplantation of MSCs. Despite of these findings above, intraventricular transplantation is quite invasive procedure requiring extreme precaution including infection prevention. When considering the simultaneous bacteremia causing meningitis, the less invasive intravenous administration of MSCs might be more clinically relevant and beneficial in meningitis with bacteremia. Further studies are necessary to confirm the therapeutic efficacy of less invasive routes, such as intraspinal or intranasal, for transplantation of MSCs in neonatal meningitis.

In our previous study of newborn brain hemorrhage model, the optimal transplantation timing generated best therapeutic effects was early peak phase of inflammation. ${ }^{40}$ In this study, MSC transplantation was delayed for $6 \mathrm{~h}$ after bacterial inoculation to allow the CSF bacterial count to reach $>1 \times 10^{5} \mathrm{CFU} / \mathrm{mL}$, which is the critical bacterial concentration sufficient to evoke meningitis inflammation. ${ }^{15,41}$ At $24 \mathrm{~h}$ after meningitis induction, CSF was obtained before introduction of antibiotics and the CSF bacterial concentration was well above $1 \times 10^{5} \mathrm{CFU} / \mathrm{mL}$ despite the significant antimicrobial effects of MSCs transplanted at $6 \mathrm{~h}$ after meningitis. This finding may suggest that MSC was administered at a time-point after meningitis had completely manifested. Therefore, there was sufficient time for the induction of inflammatory responses and meningitis-induced brain damage and for the protective and antimicrobial effects of MSCs to be exerted before antibiotic treatment.

The limitations of the present study stem from the lack of longterm follow up outcomes including hearing ability to confirm whether the protective effects of MSC transplantation against neonatal meningitis persist into adulthood. Having proven the therapeutic efficacy of MSC transplantation in this study, further studies to determine the optimal dose and therapeutic time window of MSC transplantation are necessary to optimize the therapeutic effects of MSC transplantation against neonatal bacterial meningitis. Furthermore, for the next study, immunohistochemistry analysis needs to be performed in a more stereologically sound way than this present study.

In summary, intraventricular transplantation of human UCBderived MSCs significantly reduced the CSF bacterial burden; improved hydrocephalus; improved performance in the negative geotaxis test; and reduced increased the inflammatory cytokines, TUNEL, GFAP, and ED-1 positive cells that were induced by $\mathrm{K} 1$ (-) $E$. coli meningitis without concomitant bacteremia, but with antibiotic treatment in newborn rats. These findings support the view that human UCB-derived MSC transplantation might be a promising new and effective adjuvant therapy in addition to antibiotics to treat neonatal bacterial meningitis.

\section{ACKNOWLEDGEMENTS}

We are grateful to Ye Rim Park, So Yoon Joo, and You Jin Chang for their technical assistance and animal modeling, and also thank Geun Ho Lim for performing copious MRI scanning work. This work was supported by a grant from the Korea Health Technology R\&D Project through the Korea Health Industry Development Institute (KHIDI), funded by the Ministry of Health \& Welfare, Republic of Korea (HR14C0008), and the Basic Science Research Program through the National Research Foundation of Korea (NRF) funded by the Ministry of Education, Science and Technology (NRF2017R1D1A1B03035528, NRF-2017R1A2B2011383).

\section{ADDITIONAL INFORMATION}

The online version of this article (https://doi.org/10.1038/s41390-018-0142-5) contains supplementary material, which is available to authorized users.

Competing interests: W.S.P. and Y.S.C. declare potential conflicts of interest arising from a filed or issued patent titled "Composition for treating inflammatory brain disease, which includes stem cell as active ingredient" as co-inventors, not as patentees.

Publisher's note: Springer Nature remains neutral with regard to jurisdictional claims in published maps and institutional affiliations.

\section{REFERENCES}

1. Schmidt-Hieber, M., Zweigner, J., Uharek, L., Blau, I. W. \& Thiel, E. Central nervous system infections in immunocompromised patients: update on diagnostics and therapy. Leuk. Lymphoma 50, 24-36 (2009).

2. Okike, I. O. et al. Incidence, aetiology and outcome of bacterial meningitis in infants aged $<90$ days in the UK and Republic of Ireland: prospective, enhanced, national population-based surveillance. Clin. Infect. Dis. 59, e150 (2014).

3. Furyk, J. S., Swann, O. \& Molyneux, E. Systematic review: neonatal meningitis in the developing world. Trop. Med Int Health 16, 672-9 (2011).

4. Kim, K. S. Pathogenesis of bacterial meningitis: from bacteraemia to neuronal injury. Nat. Rev. Neurosci. 4, 376-85 (2003).

5. Ahn, S. Y. et al. Long-term (postnatal day 70 ) outcome and safety of intratracheal transplantation of human umbilical cord blood-derived mesenchymal stem cells in neonatal hyperoxic lung injury. Yonsei Med J. 54, 416-24 (2013).

6. van Haaften, T. et al. Airway delivery of mesenchymal stem cells prevents arrested alveolar growth in neonatal lung injury in rats. Am. J. Respir. Crit. Care Med. 180, 1131-42 (2009).

7. Aslam, M. et al. Bone marrow stromal cells attenuate lung injury in a murine model of neonatal chronic lung disease. Am. J. Respir. Crit. Care Med. 180, 1122-30 (2009).

8. Park, W. S. et al. Hypothermia augments neuroprotective activity of mesenchymal stem cells for neonatal hypoxic-ischemic encephalopathy. PLOS ONE 10, e0120893 (2015).

9. Lee, J. A. et al. Mesenchymal stem-cell transplantation for hypoxic-ischemic brain injury in neonatal rat model. Pediatr. Res. 67, 42-6 (2010).

10. Kim, E. S. et al. Human umbilical cord blood-derived mesenchymal stem cell transplantation attenuates severe brain injury by permanent middle cerebral artery occlusion in newborn rats. Pediatr. Res. 72, 277-84 (2012).

11. van Velthoven, C. T. et al. Mesenchymal stem cell transplantation attenuates brain injury after neonatal stroke. Stroke 44, 1426-32 (2013).

12. Ahn, S. Y. et al. Mesenchymal stem cells prevent hydrocephalus after severe intraventricular hemorrhage. Stroke 44, 497-504 (2013).

13. Krasnodembskaya, A. et al. Human mesenchymal stem cells reduce mortality and bacteremia in gram-negative sepsis in mice in part by enhancing the phagocytic activity of blood monocytes. Am. J. Physiol. Lung Cell Mol. Physiol. 302, L1003-13 (2012).

14. Sung, D. K. et al. Antibacterial effect of mesenchymal stem cells against Escherichia coli is mediated by secretion of beta- defensin- 2 via toll- like receptor 4 signalling. Cell Microbiol. 18, 424-36 (2016).

15. Kim, K. S. et al. The K1 capsule is the critical determinant in the development of Escherichia coli meningitis in the rat. J. Clin. Invest 90, 897-905 (1992).

16. Chua, C. Neonatal meningitis and ventriculitis. J. Natl. Med Assoc. 70, 794-5 (1978)

17. Brandt, C. T. et al. In vivo study of experimental pneumococcal meningitis using magnetic resonance imaging. BMC Med Imaging 8, 1 (2008).

18. Jin, H. J. et al. Comparative analysis of human mesenchymal stem cells from bone marrow, adipose tissue, and umbilical cord blood as sources of cell therapy. Int J. Mol. Sci. 14, 17986-18001 (2013).

19. Ahn, S. Y. et al. Cell type-dependent variation in paracrine potency determines therapeutic efficacy against neonatal hyperoxic lung injury. Cytotherapy 17, 1025-35 (2015).

20. Shah, D. K., Daley, A. J., Hunt, R. W., Volpe, J. J. \& Inder, T. E. Cerebral white matter injury in the newborn following Escherichia coli meningitis. Eur. J. Paediatr. Neurol. 9, 13-7 (2005).

21. Rodriguez-Fanjul, J. et al. Neuroprotection with hypothermia and allopurinol in an animal model of hypoxic-ischemic injury: Is it a gender question? PLOS ONE 12, e0184643 (2017).

22. Romijn, H. J., Hofman, M. A. \& Gramsbergen, A. At what age is the developing cerebral cortex of the rat comparable to that of the full-term newborn human baby? Early Hum. Dev. 26, 61-7 (1991).

23. Polin, R. A. \& Harris, M. C. Neonatal bacterial meningitis. Semin Neonatol. 6, 157-72 (2001). 
24. Krasnodembskaya, A. et al. Antibacterial effect of human mesenchymal stem cells is mediated in part from secretion of the antimicrobial peptide LL-37. Stem Cells 28, 2229-38 (2010)

25. Waterman, R. S., Tomchuck, S. L., Henkle, S. L. \& Betancourt, A. M. A new mesenchymal stem cell (MSC) paradigm: polarization into a pro-inflammatory MSC1 or an Immunosuppressive MSC2 phenotype. PLOS ONE 5, e10088 (2010).

26. Ahn, S. Y. et al. Pivotal role of brain-derived neurotrophic factor secreted by mesenchymal stem cells in severe intraventricular hemorrhage in newborn rats. Cell Transplant. 26, 145-56 (2017).

27. Chang, Y. S. et al. Intratracheal transplantation of human umbilical cord bloodderived mesenchymal stem cells dose-dependently attenuates hyperoxiainduced lung injury in neonatal rats. Cell Transplant. 20, 1843-54 (2011).

28. Wannemuehler, T. J. et al. Advances in mesenchymal stem cell research in sepsis. J. Surg. Res. 173, 113-26 (2012).

29. Zhu, Y. G. et al. Human mesenchymal stem cell microvesicles for treatment of escherichia coli endotoxin-induced acute lung injury in mice. Stem Cells 32, 116-25 (2014).

30. Gupta, N. et al. Mesenchymal stem cells enhance survival and bacterial clearance in murine Escherichia coli pneumonia. Thorax 67, 533-9 (2012).

31. Wang, Z. J. et al. Lipopolysaccharides can protect mesenchymal stem cells (MSCs) from oxidative stress-induced apoptosis and enhance proliferation of MSCs via Toll-like receptor(TLR)-4 and PI3K/Akt. Cell Biol. Int. 33, 665-74 (2009).

32. Sporrborn, J. L. et al. Brain ventricular dimensions and relationship to outcome in adult patients with bacterial meningitis. BMC Infect. Dis. 15, 367 (2015)

33. Grandgirard, D., Steiner, O., Tauber, M. G. \& Leib, S. L. An infant mouse model of brain damage in pneumococcal meningitis. Acta Neuropathol. 114, 609-17 (2007).

34. Gianinazzi, C. et al. Caspase-3 mediates hippocampal apoptosis in pneumococcal meningitis. Acta Neuropathol. 105, 499-507 (2003).

35. Hobbs, C. et al. Xenon and hypothermia combine additively, offering long-term functional and histopathologic neuroprotection after neonatal hypoxia/ischemia. Stroke 39, 1307-13 (2008).
36. Daum, R. S., Scheifele, D. W., Syriopoulou, V. P., Averill, D. \& Smith, A. L. Ventricular involvement in experimental Hemophilus influenzae meningitis. J. Pediatr. 93, 927-30 (1978).

37. Gilles, F. H., Jammes, J. L. \& Berenberg, W. Neonatal meningitis. The ventricle as a bacterial reservoir. Arch. Neurol. 34, 560-2 (1977).

38. Friede, R. L. Cerebral infarcts complicating neonatal leptomeningitis. Acute and residual lesions. Acta Neuropathol. 23, 245-53 (1973).

39. Ahn, S. Y. et al. Optimal route for mesenchymal stem cells transplantation after severe intraventricular hemorrhage in newborn rats. PLOS ONE 10, e0132919 (2015).

40. Park, W. S. et al. Optimal timing of mesenchymal stem cell therapy for neonatal intraventricular hemorrhage. Cell Transplant. 25, 1131-44 (2016).

41. La Scolea, L. J. Jr \& Dryja, D. Quantitation of bacteria in cerebrospinal fluid and blood of children with meningitis and its diagnostic significance. J. Clin. Microbiol. 19, 187-90 (1984).

Open Access This article is licensed under a Creative Commons Attribution 4.0 International License, which permits use, sharing, adaptation, distribution and reproduction in any medium or format, as long as you give appropriate credit to the original author(s) and the source, provide a link to the Creative Commons license, and indicate if changes were made. The images or other third party material in this article are included in the article's Creative Commons license, unless indicated otherwise in a credit line to the material. If material is not included in the article's Creative Commons license and your intended use is not permitted by statutory regulation or exceeds the permitted use, you will need to obtain permission directly from the copyright holder. To view a copy of this license, visit http://creativecommons. org/licenses/by/4.0/.

(c) The Author(s) 2018 